\title{
Exception for the zero-forward-scattering theory
}

\author{
B. García-Cámara,* F. González, F. Moreno, and J. M. Saiz \\ Grupo de Óptica, Departamento de Física Aplicada, Universidad de Cantabria, Avda. de los Castros s/n, \\ 39005 Santander, Spain \\ *Corresponding author: garciacb@unican.es
}

Received August 12, 2008; accepted September 18, 2008; posted September 26, 2008 (Doc. ID 99984); published October 30, 2008

\begin{abstract}
Studies on single scattering of electromagnetic waves by magnetic particles were reported in the 1980s by Kerker et al. [J. Opt. Soc. Am. 73, 765 (1983)]. They obtained that very small spherical particles with electric permittivity and magnetic permeability values such that $\varepsilon=(4-\mu) /(2 \mu+1)$ do not produce forward scattering. We show here that this condition contains an interesting exception at $(\varepsilon=-2, \mu=-2)$ when electric and magnetic resonances are present and around which the scattered field distribution is computed and described showing a polarization-insensitive behavior at the point $(\varepsilon=-2, \mu=-2)$. () 2008 Optical Society of America OCIS codes: $290.2558,290.4020,160.1245$.
\end{abstract}

\section{INTRODUCTION}

The analysis of light scattering by particles is an important technique with applications in several fields such as atmospheric contamination, biology, and medicine (as the basis of some biosensors and proposed treatments), and even in industry, for example to detect contaminants on the surface of silicon wafers. The first formal study of this problem was carried out by Mie a century ago, considering a homogeneous sphere of arbitrary size [1]. New advances in materials technology have expanded the role of particles on the nanometric scale. Light scattering by particles smaller or much smaller than the incident wavelength (i.e., nanoparticles in the visible range) has attracted the interest of researchers in the field of nanotechnology and constitutes an important part of the field of nanophotonics. Probably the most important reason for the interest in nanoparticles lies in the possibility of exciting resonances and, more particularly, those appearing in metallic nanoparticles [localized surface plasmon resonances (LSPR)]. The main consequence is the enhancement of the local electromagnetic field, with a spatial distribution that is strongly dependent on the mode of the LSPR excited. Such a local enhancement has important applications in the biomedical sciences (biosensing, nanorulers, molecular orientation sensing, etc.) [2] and in industry (light-guiding communications, enhancement of efficient performance of solar cells, etc.) [3]. In recent years, numerous studies have been published on isolated metallic nanoparticles, dimmers, aggregates, and nanoparticles above flat substrates [4-6]. Also, with the recent appearance of engineered materials (known as "metamaterials") whose optical properties can be controlled, some anomalous, though very interesting, properties of the light scattering have been observed. There are several studies on light scattering by small particles with double negative optical properties $(\varepsilon<0$ and $\mu<0)$ [7-9]; some of them propose applications of these systems, such as, for example, nanocircuits for optical communications or optical computing based on metamaterials [10]. Some of the pioneers were Kerker et al., who studied light scattering by small particles with various values of $\varepsilon$ and $\mu$ [11]. One of the most important conclusions of their work is that under a certain condition for the optical constants, light scattering in the forward direction is zero. Some authors have tried to show experimentally zero forward scattering using Kerker's theory [12]. But this theory must be used carefully because it presents important exceptions [13]. The objective of this research is to show that the zero-forward-scattering condition, proposed by Kerker et al. presents an exception when electric and magnetic resonances are excited simultaneously.

\section{KERKER'S THEORY}

Kerker et al. [11] made a formal study of some interesting features of light scattering by small, magnetic, spherical particles using an approximation of Mie theory. This approximation can be called the "small-sphere" approximation, and it assumes that the particle has a behavior similar to that of a dipole. Under this approximation two conditions must be fulfilled: (i) $x \ll 1$ and (ii) $|m| x \ll 1$, where $m$ is the refractive index of the particle relative to the surrounding medium and $x$ is the size parameter defined as $x=2 \pi R / \lambda$, where $R$ is the radius of the sphere and $\lambda$ the incident wavelength [14]. When these two conditions are satisfied, the first two terms of the Mie expansion are a good approximation of the light scattering; that is, all coefficients of order higher than $a_{1}$ and $b_{1}$ can be neglected. The scattered intensity can be expressed in these conditions as [14]

$$
\begin{array}{l|l|l}
I_{1}=\frac{\lambda^{2}}{4 \pi^{2} r^{2}} \mid \frac{3}{2}\left(a_{1} \pi_{1}(\cos \theta)+b_{1} \tau_{1}(\cos \theta)\right. & \left.\right|^{2} \sin ^{2} \phi, \\
I_{2}=\frac{\lambda^{2}}{4 \pi^{2} r^{2}} \mid \frac{3}{2}\left(a_{1} \tau_{1}(\cos \theta)+b_{1} \pi_{1}(\cos \theta)\right. & \left.\right|^{2} \sin ^{2} \phi,
\end{array}
$$

$r$ being the distance to the observer, $a_{1}$ and $b_{1}$ the first two Mie coefficients, $\pi_{1}$ and $\tau_{1}$ the angular functions of 


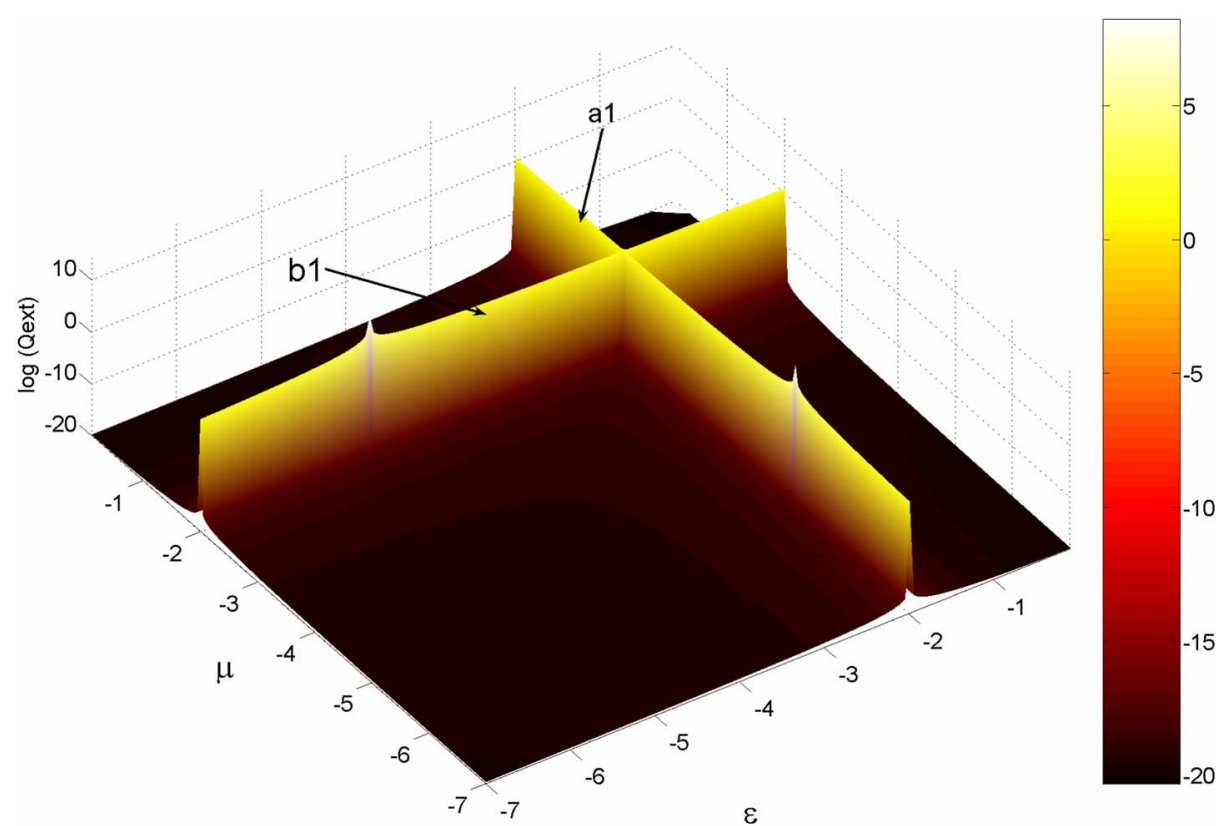

Fig. 1. (Color online) 3D plot of $\log \left(Q_{\text {ext }}\right)$ as a function of the optical properties $(\varepsilon$ and $\mu)$ in the negative-negative range for a spherical particle of $R=10^{-6} \lambda$.

first order described in [14], $\theta$ the scattering angle, $\phi$ the angle between the incident electric field and the scattering plane, and $I_{1}$ and $I_{2}$ the intensity associated with the polarized components: electric vector perpendicular and parallel to the scattering plane, respectively.

Under the small-sphere approximation, the expressions of the Mie coefficients can be also simplified in the following way:

$$
\begin{aligned}
& a_{1}=\frac{2 i}{3} x^{3}\left(\frac{\varepsilon-1}{\varepsilon+2}\right), \\
& b_{1}=\frac{2 i}{3} x^{3}\left(\frac{\mu-1}{\mu+2}\right) .
\end{aligned}
$$

If we substitute these expressions and those of the angular functions of first degree $\pi_{1}$ and $\tau_{1}$, the scattered intensity from a Rayleigh spherical particle is

$$
\begin{aligned}
& I_{1}=\frac{\lambda^{2}}{4 \pi^{2} r^{2}} x^{6}\left|\left(\frac{\varepsilon-1}{\varepsilon+2}\right)+\left(\frac{\mu-1}{\mu+2}\right) \cos (\theta)\right|^{2} \sin ^{2} \phi, \\
& I_{2}=\frac{\lambda^{2}}{4 \pi^{2} r^{2}} x^{6}\left|\left(\frac{\varepsilon-1}{\varepsilon+2}\right) \cos (\theta)+\left(\frac{\mu-1}{\mu+2}\right)\right|^{2} \sin ^{2} \phi .
\end{aligned}
$$

Kerker et al. [11] established from these expressions that when $\varepsilon=\mu$ the scattering intensity is zero in the backward direction $\left(\theta=180^{\circ}\right)$. In the forward direction $\left(\theta=0^{\circ}\right)$ the scattering should also be zero if the optical constants verify the relation

$$
\varepsilon=\frac{4-\mu}{2 \mu+1} .
$$

Under this condition $a_{1}=-b_{1}$, so the effect of the electric term $\left(a_{1}\right)$ on the scattering compensates the effect of the magnetic one $\left(b_{1}\right)$.

\section{RESULTS}

However, the expressions for the Mie coefficients given by Eqs. (2) present a singularity at $\varepsilon=-2$ and $\mu=-2$. For these values the scattering coefficients $a_{1}$ and $b_{1}$ tend to infinity, corresponding to the excitation of a Mie resonance. The electric dipolar resonance appears at $\varepsilon=-2$ and the dipolar magnetic mode at $\mu=-2$, as can be seen in Fig. 1, where we plot the extinction efficiency on the semi$\log$ axis for an isolated sphere with $R=10^{-6} \lambda$ and for $\varepsilon$ $<0$ and $\mu<0$. Dipolar electric and magnetic resonances appear as two straight branches coinciding at the point $(\varepsilon=-2, \mu=-2)$.

The singularity of this point $(\varepsilon=-2, \mu=-2)$ lies in the fact that it verifies both the zero-backscattering $(\varepsilon=\mu)$ and zero-forward-scattering [Eq. (4)] conditions, as proposed by Kerker et al. [11]. However, at this point the electric and the magnetic effects cannot be compensated, as occurs in other cases, and forward scattering is nonzero. This is because two Mie resonances (electric and magnetic) appear simultaneously in the small-sphere limit when $\varepsilon=\mu=-2$. In Fig. 2 we show the scattering patterns for a very small sphere with radius $R=10^{-6} \lambda$ and optical constants $(\varepsilon=-2, \mu=-2)$. As can be seen, the backward scattering is null, but the scattering in the forward direction acquires important values, even when Kerker's condition for zero forward scattering is fulfilled. So this point can be considered an exception to the zero-forwardscattering condition, to add to the trivial exception $(\varepsilon=\mu$ $=1$ ).

In Fig. 3 we show the scattering diagrams for a particle with $R=10^{-6} \lambda$ and for several pairs of values $(\varepsilon, \mu)$ neighboring the exception $(-2,-2)$ and verifying Eq. (4) for zero 


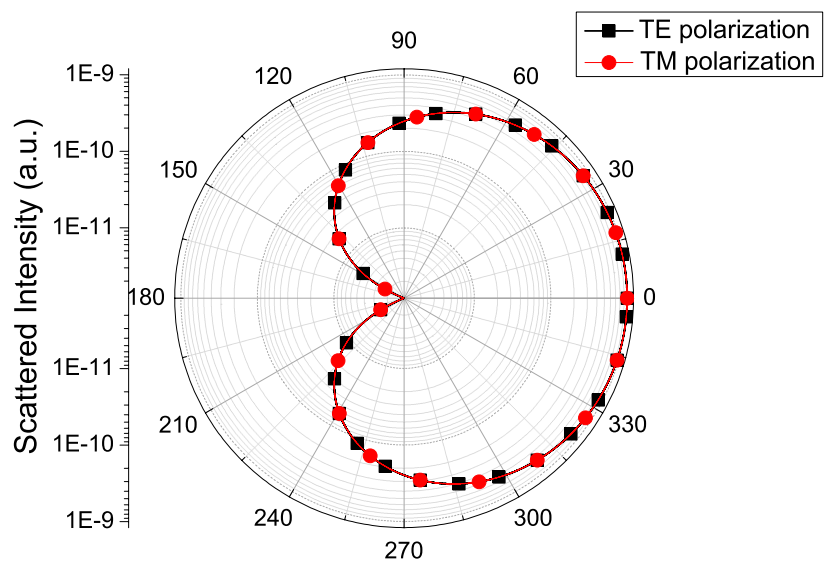

Fig. 2. (Color online) Scattering diagram for a very small particle $\left(R=10^{-6} \lambda\right)$ with optical properties $(\varepsilon=\mu=-2)$ and for both incident polarizations: TE and TM. forward scattering. The polar plots of the scattering are shown for (a) TM incident polarization and (b) TE polarization. With this figure we want to show that the exception occurs only at this point. Even for pairs of values very close to $(-2,-2)$ the scattering in the forward direction is zero as long as the condition of Eq. (4) is satisfied. We should add that the scale of the scattering for the singular point $(-2,-2)$ is huge compared with the rest of the pairs that are out of the branches of resonance of Fig. 1.

As can be seen in Fig. 3, the spatial distribution of the scattered intensity is identical for the two polarizations. This occurs because under the condition of Eq. (4), the electric and the magnetic contributions to the scattering are the same, and the effect of the switch from TM to TE has no effect on the overall scattering. Obviously, when Eq. (4) is not fulfilled, the spatial distributions produced by orthogonal incident polarizations differ completely. As

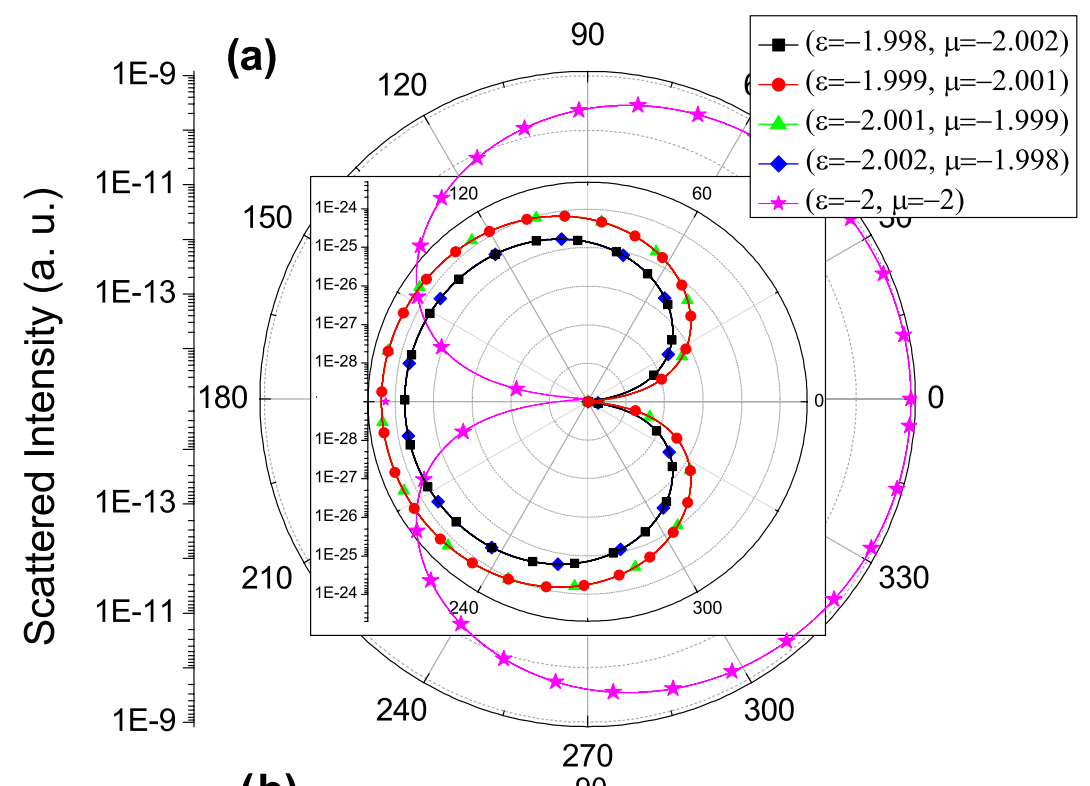

(b)

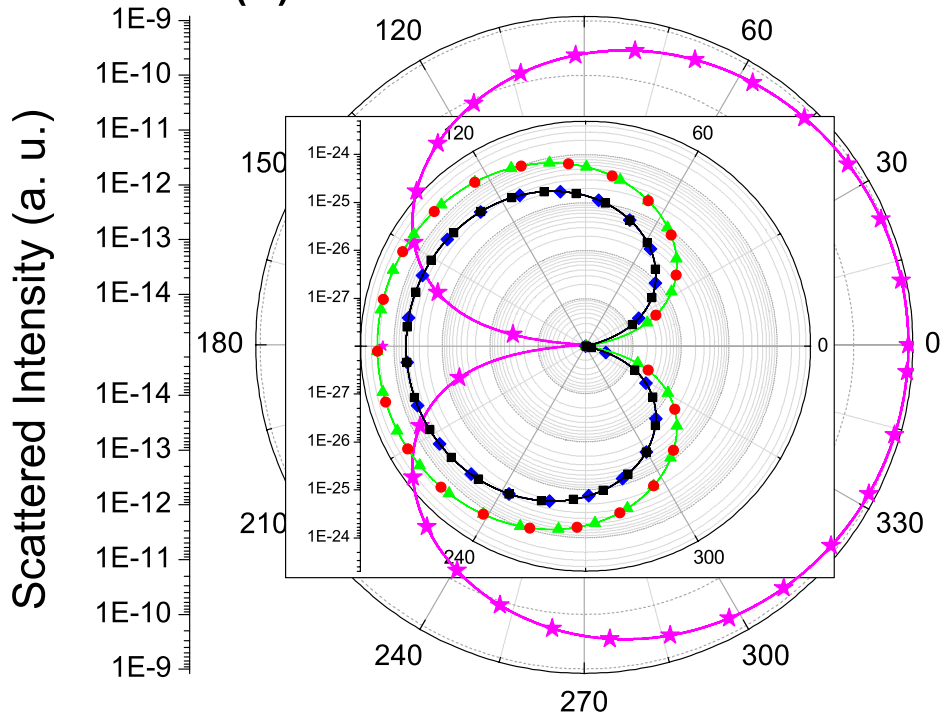

Fig. 3. (Color online) Scattering diagram for a very small particle $\left(R=10^{-6} \lambda\right)$ for both incident polarizations: (a) TM and (b) TE and for several pairs of values of the optical constants around $(\varepsilon=-2, \mu=-2)$ that verify Kerker's condition for zero forward scattering. The inset scale is for all pairs $(\varepsilon, \mu)$ diffferent for $(-2,-2)$, the latter requiring the left general scale, which is huge in comparison with the rest. 

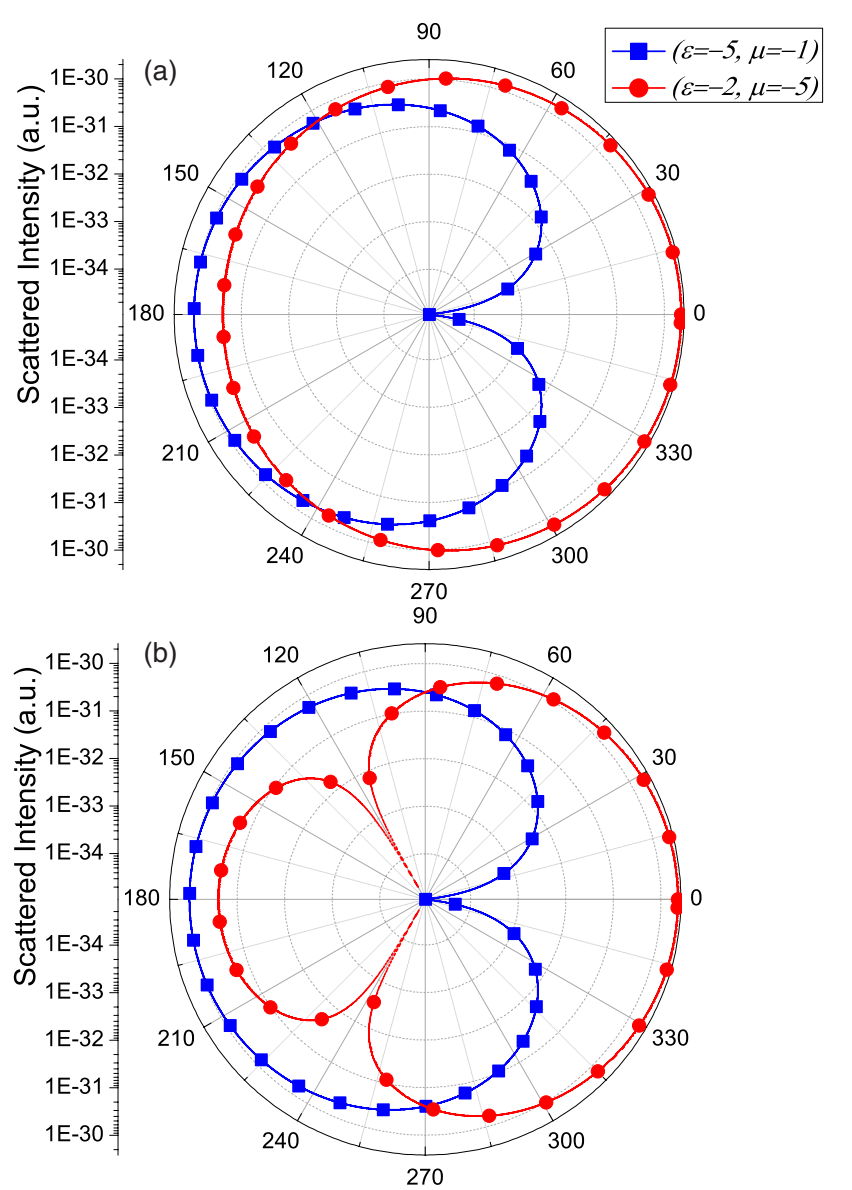

Fig. 4. (Color online) Scattering diagrams for a particle with $R=10^{-6} \lambda$ for both incident polarizations: (a) TM and (b) TE and for optical constants under (squares) or out of (circles) Kerker's condition.

an example, we show in Fig. 4 the scattering diagrams for two cases out of resonance: under Kerker's condition ( $\varepsilon=-5, \mu=-1$, squares) and out of this condition $(\varepsilon=-3$, $\mu=-5$, circles).

\section{CONCLUSIONS}

We have shown the existence of an exception to the zeroforward-scattering condition proposed by Kerker et al. [11] at $(\varepsilon=-2, \mu=-2)$ resulting from the excitation of two dipolar resonances, an electric one due to the maximum value of coefficient $a_{1}$ and a magnetic one due to the maximum value of Mie coefficient $b_{1}$. This excitation can be seen mathematically in the expressions for the first two Mie coefficients under the small-sphere approximation. Furthermore, this point verifies Kerker's condition for zero backscattering because the values of the optical constants are equal; and in this case the scattering patterns confirm this zero, showing also a TM-TE identity charac- teristic of the zero-forward-scattering condition in the small-sphere regime. In this context, this kind of study helps us to understand the unusual properties and angular response of new engineered materials. Also, it is a new step to show us the ability of these materials to govern the directionality of scattered beams at the nanoscale. Thus the new engineered materials have promising potential for configuration design in nanocircuits, light guides, etc.

\section{ACKNOWLEDGMENTS}

This research was carried out with the financial support of the Ministry of Education of Spain under project FIS2007-60158. Braulio García-Cámara thanks University of Cantabria for his Ph.D. grant.

\section{REFERENCES}

1. G. Mie, "Beiträge zur Optik trüber Medien, speziell kolloidaler Metallösungen," Ann. Phys. 330, 377-445 (1908).

2. C. Hägglund, M. Zäch, and B. Kasemo, "Enhanced charge carrier generation in dye sensitized solar cells by nanoparticle plasmons," Appl. Phys. Lett. 92, 013113 (2008).

3. W. Yan, X. Feng, X. Chen, W. Hou, and J.-J. Zhu, "A super highly sensitive glucose biosensor based on $\mathrm{Au}$ nanoparticles-AgCl@polyaniline hybrid material,” Biosens. Bioelectron. 23, 925-931 (2008).

4. G. Videen and W. S. Bickel, "Light scattering resonance in small spheres," Phys. Rev. A 45, 6008-6012 (1992).

5. F. Moreno, F. González, and J. M. Saiz, "Plasmon spectroscopy of metallic nanoparticles above flat dielectric substrates," Opt. Lett. 31, 1902-1904 (2006).

6. O. Merchiers, F. Moreno, F. González, J. M. Saiz, and G. Videen, "Electromagnetic wave scattering from two interacting small spherical particles. Influence of their optical constants, $\varepsilon$ and $\mu$," Opt. Commun. 269, 1-7 (2007).

7. C. M. Soukoulis, S. Linden, and M. Wegener, "Negative refractive index at optical wavelength," Science 315, 47-49 (2007).

8. V. Shalaev, "Optical negative-index metamaterial," Nat. Photonics 1, 41-48 (2007).

9. B. García-Cámara, F. Moreno, F. González, J. M. Saiz, and G. Videen, "Light scattering resonances by small particles with electric and magnetic properties," J. Opt. Soc. Am. A 25, 327-334 (2008).

10. N. Engheta, "Circuits with light at nanoscales: optical nanocircuits inspired by metamaterials," Science 317, 1698-1702 (2007).

11. M. Kerker, D.-S. Wang, and C. L. Giles, "Electromagnetic scattering by magnetic spheres," J. Opt. Soc. Am. 73, 765-767 (1983).

12. R. V. Metha, R. Patel, R. Desai, R. V. Upadhayay, and K. Parekh, "Experimental evidence of zero forward scattering by magnetic spheres," Phys. Rev. Lett. 96, 127402 (2006).

13. B. García-Cámara, F. Moreno, F. González, and J. M. Saiz, "Comment on 'Experimental evidence of zero forward scattering by magnetic spheres'," Phys. Rev. Lett. 98, 179701 (2007).

14. C. Bohren and D. Huffman, Absorption and Scattering of Light by Small Particles (Wiley-Interscience, 1983). 